\title{
Positivity of iterated sequences of polynomials *
}

\author{
Bao-Xuan Zhu \\ School of Mathematics and Statistics, Jiangsu Normal University, Xuzhou 221116, PR China
}

\begin{abstract}
In this paper, we present some criteria for the 2-q-log-convexity and 3-q-logconvexity of combinatorial sequences, which can be regarded as the first column of certain infinite triangular array $\left[A_{n, k}(q)\right]_{n, k \geq 0}$ of polynomials in $q$ with nonnegative coefficients satisfying the recurrence relation

$$
A_{n, k}(q)=A_{n-1, k-1}(q)+g_{k}(q) A_{n-1, k}(q)+h_{k+1}(q) A_{n-1, k+1}(q) .
$$

Those criterions can also be presented by continued fractions and generating functions. These allow a unified treatment of the 2- $q$-log-convexity of alternating Eulerian polynomials, 2-log-convexity of Euler numbers, and 3- $q$-log-convexity of many classical polynomials, including the Bell polynomials, the Eulerian polynomials of Types $A$ and $B$, the $q$-Schröder numbers, $q$-central Delannoy numbers, the Narayana polynomials of Types $A$ and $B$, the generating functions of rows in the Catalan triangles of Aigner and Shapiro, the generating functions of rows in the large Schröder triangle, and so on, which extend many known results for $q$-log-convexity.
\end{abstract}

MSC: 05A20; 05A30; 11B37; 11B83; 30B70

Keywords: Log-convexity; $q$-log-convexity; $k$ - $q$-log-convexity; $r$-Order total positivity; Continued fractions

\section{Introduction}

\section{$1.1 \quad$ Notation}

Let $\left\{a_{n}\right\}_{n \geq 0}$ be a sequence of nonnegative real numbers. The sequence is called $\log$ concave (resp. log-convex) if for all $n \geq 1, a_{n-1} a_{n+1} \leq a_{n}^{2}$ (resp. $a_{n-1} a_{n+1} \geq a_{n}^{2}$ ). The log-convex and log-concave sequences arise often in combinatorics, algebra, geometry, analysis, probability and statistics and have been extensively investigated, see Stanley [41] and Brenti [10] for log-concavity, Liu and Wang [30] for log-convexity.

In recent years there has been a growing interest in the $q$-log-concavity and q-logconvexity of $q$-analogs of combinatorial sequences. Recall their definitions. For two

${ }^{*}$ Supported partially by the National Natural Science Foundation of China (No. 11571150).

Email address: bxzhu@jsnu.edu.cn (B.-X. Zhu) 
polynomials with real coefficients $f(q)$ and $g(q)$, denote $f(q) \geq_{q} g(q)$ if the difference $f(q)-g(q)$ has only nonnegative coefficients. For a polynomial sequence $\left\{f_{n}(q)\right\}_{n \geq 0}$, it is called $q$-log-concave suggested by Stanley if

$$
f_{n}(q)^{2} \geq_{q} f_{n+1}(q) f_{n-1}(q)
$$

for $n \geq 1$. It is called $q$-log-convex defined by Liu and Wang if

$$
f_{n+1}(q) f_{n-1}(q) \geq_{q} f_{n}(q)^{2}
$$

for $n \geq 1$. It was proved that many famous polynomials have the $q$-log-concavity or the $q$-log-convexity, e.g., $q$-binomial coefficients and $q$-Stirling numbers of two kinds [13, 28, 37], the Bell polynomials [30], the classical Eulerian polynomials [30, 50], the Narayana polynomials of type $A$ [17], the Narayana polynomials of type $B[18,50]$, the generating functions of Jacobi-Stirling numbers [29, 51], the Bessel polynomials [19], the Ramanujan polynomials [19], and so on.

Motivated by the notion of infinite log-concavity [32] and infinite $q$-log-concavity [33], as an extension of $q$-log-convexity, Chen [15] defined the infinite $q$-log-convexity as follows. Define the operator $\mathcal{L}$ which maps a polynomial sequence $\left\{f_{i}(q)\right\}_{i \geq 0}$ to a polynomial sequence $\left\{g_{i}(q)\right\}_{i \geq 1}$ given by

$$
g_{i}(q):=f_{i-1}(q) f_{i+1}(q)-f_{i}(q)^{2} .
$$

Then the $q$-log-convexity of $\left\{f_{i}(q)\right\}_{i \geq 0}$ is equivalent to the $q$-positivity of $\mathcal{L}\left\{f_{i}(q)\right\}$, i.e., the coefficients of $g_{i}(q)$ are nonnegative for all $i \geq 1$. In general, we say that $\left\{f_{i}(q)\right\}_{i \geq 0}$ is $k$-q-log-convex if the coefficients of $\mathcal{L}^{m}\left\{f_{i}(q)\right\}$ are nonnegative for all $m \leq k$, where $\mathcal{L}^{m}=\mathcal{L}\left(\mathcal{L}^{m-1}\right)$. It is called infinitely $q$-log-convex if $\left\{f_{i}(q)\right\}_{i \geq 0}$ is $k$ - $q$-log-convex for every $k \geq 0$. If we take $q=0$, then the polynomial sequence reduces to a sequence of real numbers. So the $k$ - $q$-log-convexity turns out to be $k$-log-convexity, see Chen and Xia [21].

\subsection{Motivations}

In [7], Boros and Moll studied the quartic integral

$$
\int_{0}^{\infty} \frac{1}{\left(t^{4}+2 x t^{2}+1\right)^{n+1}} d t
$$

and got the following formula for any $x>-1$ and any nonnegative integer $n$ that

$$
\int_{0}^{\infty} \frac{1}{\left(t^{4}+2 x t^{2}+1\right)^{n+1}} d t=\frac{\pi}{2^{n+3 / 2}(x+1)^{n+1 / 2}} P_{n}(x),
$$

where

$$
P_{n}(x)=\sum_{j, k}\left(\begin{array}{c}
2 n+1 \\
2 j
\end{array}\right)\left(\begin{array}{c}
n-j \\
k
\end{array}\right)\left(\begin{array}{c}
2 k+2 j \\
k+j
\end{array}\right) \frac{(x+1)^{j}(x-1)^{k}}{2^{3(k+j)}}
$$

are called the Boros-Moll polynomials. Moll [32] conjectured that the coefficients of $P_{n}(x)$ form a log-concave sequence, which was proved by Kauers and Paule [27]. Moreover, as an extension, Chen et.al [20, 16] proved that 2-log-concavity and 3-log-concavity of the 
coefficients of $P_{n}(x)$ and confirmed two Brändén's conjectures on real roots of polynomials related to $P_{n}(x)$ [8]. However, the stronger conjecture on the infinitely log-concavity of the coefficients of $P_{n}(x)$ proposed by Boros and Moll [7] is still open. On the other hand, Chen [15] also proposed the next open conjecture on the infinite $q$-log-convexity.

Conjecture 1.1. Boros-Moll polynomials are infinitely q-log-convex.

In general, it is much more difficult to show the $k$ - $q$-log-convexity for $k \geq 2$. So far, there has been no nontrivial example. Thus, motivated by these, we will study the higher order $q$-log-convexity in this paper.

Let $\sigma=\left(g_{k}(q)\right)_{k \geq 0}$ and $\tau=\left(h_{k+1}(q)\right)_{k \geq 0}$ be two sequences of polynomials. Define an infinite lower triangular matrix $\left[A_{n, k}(q)\right]_{n, k \geq 0}$ satisfies the recurrence

$$
A_{0,0}(q)=1, \quad A_{n, k}(q)=A_{n-1, k-1}(q)+g_{k}(q) A_{n-1, k}(q)+h_{k+1}(q) A_{n-1, k+1}(q)
$$

where $A_{n, k}(q)=0$ unless $n \geq k \geq 0$. Many well-known polynomials can be viewed as the first column $A_{n, 0}(q)$ of $\left[A_{n, k}(q)\right]_{n, k \geq 0}$. The following are some classical examples, e.g., see $[2,48]$.

\section{Example 1.1.}

(i) If $g_{k}=k+q$ and $h_{k}=k q$, then the first column $A_{n, 0}(q)$ for $n \geq 0$ equal to the Bell polynomials, which are the generating functions $B_{n}(q)=\sum_{k=0}^{n} S(n, k) q^{k}$ of the Stirling numbers of the second kind. They can be looked as a $q$-analog of the Bell numbers and have many fascinating properties (see [36, §4.1.3] for instance). In addition, it is well known that the Bell polynomials $B_{n}(q)$ have only real zeros and $S(n, k)$ is therefore log-concave in $k$ for each fixed $n$ (see [46] for instance).

(ii) If $g_{k}=(k+1) q+k$ and $h_{k}=k^{2} q$, then the first column $A_{n, 0}(q)$ for $n \geq 0$ equal to the Eulerian polynomials defined by the descent statistics. Let $\pi=a_{1} a_{2} \cdots a_{n}$ be a permutation of $[n]$. An element $i \in[n-1]$ is called a descent of $\pi$ if $a_{i}>a_{i+1}$. Denote by $A(n, k)$ the number of permutations of $[n]$ having $k-1$ descents, which is called the Eulerian number. Then the Eulerian polynomials $E_{n}(q)=\sum_{k=0}^{n} E(n, k) q^{k}$ for $n \geq 0$. It is well known that $A_{n}(q)$ has only real zeros and $A(n, k)$ is therefore log-concave in $k$ for each fixed $n$ (see [46] for instance).

(iii) If $g_{0}=q+1, g_{k}=2 q+1$ and $h_{k}=q(q+1)$, then the first column $A_{n, 0}(q)$ for $n \geq 0$ equal to the $q$-Schröder numbers $r_{n}(q)=\sum_{k=0}^{n}\left(\begin{array}{c}n+k \\ n-k\end{array}\right) \frac{1}{k+1}\left(\begin{array}{c}2 k \\ k\end{array}\right) q^{k}$ [6]. They are defined as the $q$-analog of the large Schröder numbers $r_{n}$ :

$$
r_{n}(q)=\sum_{P} q^{\operatorname{diag}(P)}
$$

where $P$ takes over all Schröder paths from $(0,0)$ to $(n, n)$ and $\operatorname{diag}(P)$ denotes the number of diagonal steps in the path $P$.

(iv) If $g_{k}=1+2 q, h_{1}=2 q(1+q)$ and $h_{k}=q(1+q)$, then the first column $A_{n, 0}(q)$ for $n \geq 0$ equal to the $q$-central Delannoy numbers $D_{n}(q)=\sum_{k=0}^{n}\left(\begin{array}{c}n+k \\ n-k\end{array}\right)\left(\begin{array}{c}2 k \\ k\end{array}\right) q^{k}[38]$, which reduce to famous central Delannoy numbers for $q=1$. 
(v) If $g_{0}=q, g_{k}=1+q$ and $h_{k}=q$, then the first column $A_{n, 0}(q)$ for $n \geq 0$ equal to the Narayana polynomials $N_{n}(q)=\sum_{k=1}^{n} \frac{1}{n}\left(\begin{array}{l}n \\ k\end{array}\right)\left(\begin{array}{c}n \\ k-1\end{array}\right) q^{k}$, where $\frac{1}{n}\left(\begin{array}{c}n \\ k\end{array}\right)\left(\begin{array}{c}n \\ k-1\end{array}\right)$ is called the Narayana number, which is defined as the number of Dyck paths of length $2 n$ with exactly $k$ peaks. In addition, the Narayana polynomials $N_{n}(q)$ are closely related to the $q$-Schröder numbers $r_{n}(q)$ by $r_{n}(q)=N_{n}(1+q)$ [43]. It was also proved in [49] that the Narayana transformation preserves strong $q$-log-convexity of polynomials.

(vi) If $g_{k}=1+q, h_{1}=2 q$ and $h_{k}=q$ for $k>1$, then the first column $A_{n, 0}(q)$ for $n \geq 0$ equal to the Narayana polynomials $W_{n}(q)=\sum_{k=0}^{n}\left(\begin{array}{l}n \\ k\end{array}\right)^{2} q^{k}$ of type $B$. They appeared as the rank generating function of the lattice of noncrossing partitions of type $B$ on $[n]=\{1,2, \ldots, n\}[35]$ and are also the coordinator polynomial of the growth series for the classical root lattice $A_{n}[4,5]$. Moreover, the Narayana transformation of Type $B$ preserves strong $q$-log-convexity of polynomials [49].

The organization of this paper is as follows. In Section 2, we state our main results and apply total positivity of matrices to give the proof of Theorem 2.1. In Section 3, we apply Theorem 2.1 to sequences of real numbers and Riordan array. In particular, we get 3-q-log-convexity of the generating functions of rows in the Catalan triangles of Aigner and Shapiro, and the large Schröder triangle. In Section 4, we apply Theorem 2.2 to some classical polynomials in a unified manner, and get the 2-q-log-convexity of alternating Eulerian polynomials, 2-log-convexity of Euler numbers, and 3- $q$-log-convexity of many classical polynomials, including the Bell polynomials, the Eulerian polynomials of Types $A$ and $B$, the $q$-Schröder numbers, $q$-central Delannoy numbers, the Narayana polynomials of Types $A$ and $B$, and so on. These applications extend and strengthen many known results for $q$-log-convexity or log-convexity. Finally, in Section 5, as the concluding remarks, we will point out some further research problems and conjectures.

\section{Main results}

In this section, we prove the next criterions for 2- $q$-log-convexity and 3- $q$-log-convexity, respectively.

Theorem 2.1. Let $\left\{g_{n}(q)\right\}_{n \geq 0}$ and $\left\{h_{n}(q)\right\}_{n \geq 1}$ be two sequences of polynomials with nonnegative coefficients. Assume that the triangular array $\left[A_{n, k}(q)\right]_{n, k \geq 0}$ is defined in (1.1). Then we have the following:

(i) If $g_{k}(q) g_{k+1}(q) g_{k+2}(q)-h_{k+1}(q) g_{k+2}(q)-g_{k}(q) h_{k+2}(q) \geq_{q} 0$ for $k \geq 0$, then the first column sequence $\left\{A_{n, 0}(q)\right\}_{n \geq 0}$ is 2-q-log-convex.

(ii) If $g_{k}(q) g_{k+1}(q) \geq_{q} h_{k+1}(q)$ and

$$
\begin{aligned}
& g_{k}(q) g_{k+1}(q) g_{k+2}(q) g_{k+3}(q)-g_{k+2}(q) g_{k+3}(q) h_{k+1}(q)-g_{k}(q) g_{k+3}(q) h_{k+2}(q)- \\
& g_{k}(q) g_{k+1}(q) h_{k+3}(q)+h_{k+1}(q) h_{k+3}(q) \geq_{q} 0
\end{aligned}
$$

for $k \geq 0$, then the first column sequence $\left\{A_{n, 0}(q)\right\}_{n \geq 0}$ is 3-q-log-convex. 
In combinatorics, one often meets the continued fractions. In fact, the sequence $\left\{A_{n, 0}(q)\right\}_{n \geq 0}$ in (1.1) is also closely related to the famous Jacobi continued fractions. If let its generating function

$$
G(x)=\sum_{n \geq 0} A_{n, 0}(q) x^{n},
$$

then it can be expressed by the Jacobi continued fraction as follows

$$
G(x)=\frac{1}{1-s_{0} x-\frac{t_{1} x^{2}}{1-s_{1} x-\frac{t_{2} x^{2}}{1-s_{2} x-\ldots}}},
$$

see [45] or [23] for instance. So, Theorem 2.1 can also be restated by the continued fraction as follows.

Theorem 2.2. Given two sequences $\left\{g_{k}(q)\right\}_{k \geq 0}$ and $\left\{h_{k}(q)\right\}_{k \geq 1}$ of polynomials with nonnegative coefficients, let

$$
\sum_{n=0}^{\infty} T_{n}(q) x^{n}=\frac{1}{1-g_{0}(q) x-\frac{h_{1}(q) x^{2}}{1-g_{1}(q) x-\frac{h_{2}(q) x^{2}}{1-g_{2}(q) x-\ldots}}} .
$$

Then we have the following:

(i) If $g_{k}(q) g_{k+1}(q) g_{k+2}(q)-h_{k+1}(q) g_{k+2}(q)-g_{k}(q) h_{k+2}(q) \geq_{q} 0$ for $k \geq 0$, then the sequence $\left\{T_{n}(q)\right\}_{n \geq 0}$ is 2-q-log-convex.

(ii) If $g_{k}(q) g_{k+1}(q) \geq_{q} h_{k+1}(q)$ and

$$
\begin{aligned}
& g_{k}(q) g_{k+1}(q) g_{k+2}(q) g_{k+3}(q)-g_{k+2}(q) g_{k+3}(q) h_{k+1}(q)-g_{k}(q) g_{k+3}(q) h_{k+2}(q)- \\
& g_{k}(q) g_{k+1}(q) h_{k+3}(q)+h_{k+1}(q) h_{k+3}(q) \geq_{q} 0
\end{aligned}
$$

for $k \geq 0$, then the sequence $\left\{T_{n}(q)\right\}_{n \geq 0}$ is 3-q-log-convex.

\subsection{Proof of Theorem 2.1}

Total positivity of matrices plays an important role in our proof. Therefore let us recall the definition. Let $M=\left[m_{n, k}\right]_{n, k>0}$ be a matrix of real numbers. It is called totally positive ( $T P$ for short) if all its minors are nonnegative and is called $T P_{r}$ if all minors of order $\leq r$ are nonnegative. When each entry of $M$ is a polynomial in $q$ with nonnegative coefficients, then we have the similar concepts for $q$ - TP (resp. $q$ - $\mathrm{TP}_{r}$ ) if all its minors (resp. if all minors of order $\leq r$ ) are polynomials with nonnegative coefficients. Total positivity of matrices plays an important role in various branches of mathematics, statistics, probability, mechanics, economics, and computer science, see Karlin [26] and Pinkus [34] for instance. Theory of total positivity has successfully been applied to log-concavity problems in combinatorics, see Brenti $[11,12]$.

In order to present our proof, we need the following some basic results from total positivity of matrices. The first of the following two lemmas is direct by the definition and the second follows from the classic Cauchy-Binet formula. 
Lemma 2.3. A matrix is $q-T P_{r}$ if and only if its leading principal submatrices are all $q-T P_{r}$.

Lemma 2.4. If two matrices are $q-T P_{r}$, then so is their product.

Using the total positivity of matrices, we have the following criterion for higher order $q$-log-convexity.

Proposition 2.5. Let $\left\{a_{n}(q)\right\}_{n \geq 0}$ be a sequence of polynomials with nonnegative coefficients. If the Hankel matrix $\left[a_{i+j}(q)\right]_{i, j \geq 0}$ is $q$-TP $P_{r+1}$, then $\left\{a_{n}(q)\right\}_{n \geq 0}$ is r-q-log-convex for $1 \leq r \leq 3$.

Proof. For brevity, we write $a_{k}$ for $a_{k}(q)$. Note for $r=1$ that

$$
\mathcal{L}\left(a_{k}\right)=a_{k+1} a_{k-1}-a_{k}^{2}=\left|\begin{array}{cc}
a_{k-1} & a_{k} \\
a_{k} & a_{k+1}
\end{array}\right| .
$$

Thus it is obvious that $\left\{a_{n}(q)\right\}_{n \geq 0}$ is q-log-convex if the Hankel matrix $\left[a_{i+j}\right]_{i, j \geq 0}$ is q- $\mathrm{TP}_{2}$. Furthermore, for $r=2$, we get that

$$
\begin{aligned}
\mathcal{L}^{2}\left(a_{k}\right) & =\mathcal{L}\left(a_{k-1}\right) \mathcal{L}\left(a_{k+1}\right)-\left[\mathcal{L}\left(a_{k}\right)\right]^{2} \\
& =\left(a_{k+2} a_{k}-a_{k+1}^{2}\right)\left(a_{k} a_{k-2}-a_{k-1}^{2}\right)-\left(a_{k+1} a_{k-1}-a_{k}^{2}\right)^{2} \\
& =a_{k}\left(2 a_{k-1} a_{k} a_{k+1}+a_{k} a_{k+2} a_{k-2}-a_{k}^{3}-a_{k+1}^{2} a_{k-2}-a_{k-1}^{2} a_{k+2}\right) \\
& =a_{k}\left|\begin{array}{ccc}
a_{k-2} & a_{k-1} & a_{k} \\
a_{k-1} & a_{k} & a_{k+1} \\
a_{k} & a_{k+1} & a_{k+2}
\end{array}\right|
\end{aligned}
$$

which implies that if the Hankel matrix $\left[a_{i+j}(q)\right]_{i, j \geq 0}$ is $\mathrm{q}-\mathrm{TP}_{3}$ then $\left\{a_{n}(q)\right\}_{n \geq 0}$ is 2 -q-logconvex.

In the following, we proceed to consider the case for $r=3$. By (2.1), we have

$$
\begin{aligned}
\mathcal{L}^{3}\left(a_{k}\right)= & \mathcal{L}^{2}\left(a_{k-1}\right) \mathcal{L}^{2}\left(a_{k+1}\right)-\left[\mathcal{L}^{2}\left(a_{k}\right)\right]^{2} \\
= & a_{k+1} a_{k-1}\left|\begin{array}{ccc}
a_{k-1} & a_{k} & a_{k+1} \\
a_{k} & a_{k+1} & a_{k+2} \\
a_{k+1} & a_{k+2} & a_{k+3}
\end{array}\right|\left|\begin{array}{ccc}
a_{k-3} & a_{k-2} & a_{k-1} \\
a_{k-2} & a_{k-1} & a_{k} \\
a_{k-1} & a_{k} & a_{k+1}
\end{array}\right|-a_{k}^{2}\left|\begin{array}{ccc}
a_{k-2} & a_{k-1} & a_{k} \\
a_{k-1} & a_{k} & a_{k+1} \\
a_{k} & a_{k+1} & a_{k+2}
\end{array}\right|^{2} \\
= & \left(a_{k+1} a_{k-1}-a_{k}^{2}\right) \times \\
& \left(a_{k}^{2}\left|\begin{array}{cccc}
a_{k-3} & a_{k-2} & a_{k-1} & a_{k} \\
a_{k-2} & a_{k-1} & a_{k} & a_{k+1} \\
a_{k-1} & a_{k} & a_{k+1} & a_{k+2} \\
a_{k} & a_{k+1} & a_{k+2} & a_{k+3}
\end{array}\right|+\left|\begin{array}{ccc}
a_{k-3} & a_{k-2} & a_{k-1} \\
a_{k-2} & a_{k-1} & a_{k} \\
a_{k-1} & a_{k} & a_{k+1}
\end{array}\right|\left|\begin{array}{ccc}
a_{k-1} & a_{k} & a_{k+1} \\
a_{k} & a_{k+1} & a_{k+2} \\
a_{k+1} & a_{k+2} & a_{k+3}
\end{array}\right|\right) .
\end{aligned}
$$

So if the Hankel matrix $\left[a_{i+j}(q)\right]_{i, j \geq 0}$ is $\mathrm{q}-\mathrm{TP}_{4}$ then $\left\{a_{n}(q)\right\}_{n \geq 0}$ is 3 -q-log-convex. This completes the proof.

For the sequence $\left\{a_{n}(q)\right\}_{n \geq 0}$ in Proposition 2.5, if let $q=0$, then Proposition 2.5 can be reduced to the following result for sequences of real numbers.

Proposition 2.6. Let $\left\{a_{n}\right\}_{n \geq 0}$ be a sequence of nonnegative real numbers. If the Hankel matrix $\left[a_{i+j}\right]_{i, j \geq 0}$ is $T P_{r+1}$, then $\left\{a_{n}\right\}_{n \geq 0}$ is $r$-log-convex for $1 \leq r \leq 3$. 
Lemma 2.7. Given two sequences $\left\{g_{k}(q)\right\}_{k \geq 0}$ and $\left\{h_{k}(q)\right\}_{k \geq 1}$ of polynomials with nonnegative coefficients, assume that the matrix

$$
\mathcal{J}_{n}(q)=\left(J_{i, j}\right)_{0 \leq i, j \leq n-1}=\left(\begin{array}{ccccc}
g_{0}(q) & 1 & 0 & \cdots & 0 \\
h_{1}(q) & g_{1}(q) & 1 & \cdots & 0 \\
0 & h_{2}(q) & g_{2}(q) & \cdots & 0 \\
\vdots & \vdots & \vdots & \cdots & \vdots \\
0 & 0 & 0 & \cdots & g_{n-1}(q)
\end{array}\right)
$$

Then we have the following.

(i) $\mathcal{J}_{n}(q)$ is $q-T P_{3}$ if and only if

$$
g_{k}(q) g_{k+1}(q) g_{k+2}(q)-h_{k+1}(q) g_{k+2}(q)-g_{k}(q) h_{k+2}(q) \geq_{q} 0
$$

for $k \geq 0$.

(ii) $\mathcal{J}_{n}(q)$ is $q-T P_{4}$ if and only if $g_{k}(q) g_{k+1}(q) \geq_{q} h_{k+1}(q)$ and

$$
\begin{aligned}
& g_{k}(q) g_{k+1}(q) g_{k+2}(q) g_{k+3}(q)-g_{k+2}(q) g_{k+3}(q) h_{k+1}(q)-g_{k}(q) g_{k+3}(q) h_{k+2}(q) \\
& -g_{k}(q) g_{k+1}(q) h_{k+3}(q)+h_{k+1}(q) h_{k+3}(q) \geq_{q} 0 .
\end{aligned}
$$

Proof. (i) Note that $\mathcal{J}_{n}(q)$ is $q-\mathrm{TP}_{2}$ if and only if

$$
g_{k}(q) g_{k+1}(q) \geq_{q} h_{k+1}(q)
$$

for $k \geq 0$. In addition, it is not hard to find that $\mathcal{J}_{n}(q)$ is $q-\mathrm{TP}_{3}$ if and only if

$$
\begin{aligned}
& g_{k}(q) g_{k+1}(q) \geq_{q} h_{k+1}(q), \\
& g_{k}(q) g_{k+1}(q) g_{k+2}(q)-h_{k+1}(q) g_{k+2}(q)-g_{k}(q) h_{k+2}(q) \geq_{q} 0
\end{aligned}
$$

for $k \geq 0$. Note that

$$
\begin{aligned}
& g_{k}(q) g_{k+1}(q) g_{k+2}(q)-h_{k+1}(q) g_{k+2}(q)-g_{k}(q) h_{k+2}(q) \\
= & g_{k+2}(q)\left[g_{k}(q) g_{k+1}(q)-h_{k+1}(q)\right]-g_{k}(q) h_{k+2}(q) .
\end{aligned}
$$

Thus if

$$
g_{k}(q) g_{k+1}(q) g_{k+2}(q)-h_{k+1}(q) g_{k+2}(q)-g_{k}(q) h_{k+2}(q) \geq_{q} 0,
$$

then we must have

$$
g_{k}(q) g_{k+1}(q) \geq_{q} h_{k+1}(q) .
$$

So it follows from

$$
g_{k}(q) g_{k+1}(q) g_{k+2}(q)-h_{k+1}(q) g_{k+2}(q)-g_{k}(q) h_{k+2}(q) \geq_{q} 0
$$

for $k \geq 0$ that $\mathcal{J}_{n}(q)$ is $q-\mathrm{TP}_{3}$. This completes the proof of (i).

(ii) Note that $\mathcal{J}_{n}(q)$ is $q-\mathrm{TP}_{4}$ if and only if $\mathcal{J}_{n}(q)$ is $q-\mathrm{TP}_{3}$ and for all $k \geq 0$ that

$$
\begin{aligned}
& g_{k}(q) g_{k+1}(q) g_{k+2}(q) g_{k+3}(q)-g_{k+2}(q) g_{k+3}(q) h_{k+1}(q)-g_{k}(q) g_{k+3}(q) h_{k+2}(q) \\
& -g_{k}(q) g_{k+1}(q) h_{k+3}(q)+h_{k+1}(q) h_{k+3}(q) \geq_{q} 0 .
\end{aligned}
$$


Thus, if

$$
g_{k}(q) g_{k+1}(q) h_{k+3}(q)-h_{k+1}(q) h_{k+3}(q)=\left[g_{k}(q) g_{k+1}(q)-h_{k+1}(q)\right] h_{k+3}(q) \geq_{q} 0
$$

and

$$
\begin{aligned}
& g_{k}(q) g_{k+1}(q) g_{k+2}(q) g_{k+3}(q)-g_{k+2}(q) g_{k+3}(q) h_{k+1}(q)-g_{k}(q) g_{k+3}(q) h_{k+2}(q) \\
& -g_{k}(q) g_{k+1}(q) h_{k+3}(q)+h_{k}(q) h_{k+3}(q) \geq_{q} 0
\end{aligned}
$$

then we have

$$
g_{k}(q) g_{k+1}(q) g_{k+2}(q)-g_{k+2}(q) h_{k+1}(q)-g_{k}(q) h_{k+2}(q) \geq_{q} 0
$$

since

$$
\begin{aligned}
& g_{k}(q) g_{k+1}(q) g_{k+2}(q) g_{k+3}(q)-g_{k+2}(q) g_{k+3}(q) h_{k+1}(q)-g_{k}(q) g_{k+3}(q) h_{k+2}(q) \\
& -g_{k}(q) g_{k+1}(q) h_{k+3}(q)+h_{k+1}(q) h_{k+3}(q) \\
= & g_{k+3}(q)\left[g_{k}(q) g_{k+1}(q) g_{k+2}(q)-g_{k+2}(q) h_{k+1}(q)-g_{k}(q)(q) h_{k+2}(q)\right]-g_{k}(q) g_{k+1}(q) h_{k+3}(q) \\
& +h_{k+1}(q) h_{k+3}(q) .
\end{aligned}
$$

Thus, if $g_{k}(q) g_{k+1}(q) \geq_{q} h_{k+1}(q)$ and

$$
\begin{aligned}
& g_{k}(q) g_{k+1}(q) g_{k+2}(q) g_{k+3}(q)-g_{k+2}(q) g_{k+3}(q) h_{k+1}(q)-g_{k}(q)(q) g_{k+3}(q) h_{k+2}(q) \\
& -g_{k}(q) g_{k+1}(q) h_{k+3}(q)+h_{k+1}(q) h_{k+3}(q) \geq_{q} 0,
\end{aligned}
$$

then $\mathcal{J}_{n}(q)$ is $q$ - $\mathrm{TP}_{4}$. The proof of (ii) is complete.

Proof of Theorem 2.1: Assume that the matrices

$$
\mathcal{Q}_{n}(q)=\left(\begin{array}{ccccc}
A_{1,0}(q) & A_{1,1}(q) & 0 & \cdots & 0 \\
A_{2,0}(q) & A_{2,1}(q) & A_{2,2}(q) & \cdots & 0 \\
A_{3,0}(q) & A_{3,1}(q) & A_{3,2}(q) & \cdots & 0 \\
\vdots & \vdots & \vdots & \cdots & \vdots \\
A_{n, 0}(q) & A_{n, 1}(q) & A_{n, 2}(q) & \cdots & A_{n, n-1}(q)
\end{array}\right)
$$

and

$$
\mathcal{A}_{n}(q)=\left(A_{i, j}(q)\right)_{0 \leq i, j \leq n-1}=\left(\begin{array}{ccccc}
A_{0,0}(q) & 0 & 0 & \cdots & 0 \\
A_{1,0}(q) & A_{1,1}(q) & 0 & \cdots & 0 \\
A_{2,0}(q) & A_{2,1}(q) & A_{2,2}(q) & \cdots & 0 \\
\vdots & \vdots & \vdots & \cdots & \vdots \\
A_{n-1,0}(q) & A_{n-1,1}(q) & A_{n-1,2}(q) & \cdots & A_{n-1, n-1}(q)
\end{array}\right) .
$$

So by (1.1) it is easy to deduce that

$$
\mathcal{Q}_{n}(q)=\mathcal{A}_{n}(q) \mathcal{J}_{n}(q)
$$


Let

$$
\mathcal{H}=\left[A_{n+m, 0}(q)\right]_{n, m \geq 0}=\left[\begin{array}{cccc}
A_{0,0}(q) & A_{1,0}(q) & A_{2,0}(q) & \cdots \\
A_{1,0}(q) & A_{2,0}(q) & A_{3,0}(q) & \cdots \\
A_{2,0}(q) & A_{3,0}(q) & A_{4,0}(q) & \cdots \\
\vdots & \vdots & \vdots & \ddots
\end{array}\right]
$$

be the Hankel matrix of the sequence $\left\{A_{n, 0}(q)\right\}_{n \geq 0}$ and the infinite matrix

$$
\mathcal{A}(q)=\left(A_{i, j}(q)\right)=\left(\begin{array}{cccc}
A_{0,0}(q) & 0 & 0 & \cdots \\
A_{1,0}(q) & A_{1,1}(q) & 0 & \cdots \\
A_{2,0}(q) & A_{2,1}(q) & A_{2,2}(q) & \cdots \\
\vdots & \vdots & \vdots & \ddots
\end{array}\right) .
$$

Claim 2.8. If $\mathcal{J}_{n}(q)$ is $q-T P_{r}$, then the Hankel matrix $\mathcal{H}$ is $q-T P_{r}$.

Proof. If $\mathcal{J}_{n}(q)$ is $q-\mathrm{TP}_{r}$, then, by induction on $n$, we conclude that $\mathcal{A}_{n}(q)$ is $q$ - $\mathrm{TP}_{r}$ by $(2.2)$ and Lemma 2.4. So is $\mathcal{A}(q)$ by Lemma 2.3. From Aigner's Fundamental Theorem in [2], we have $\mathcal{H}=\mathcal{A}(q) \mathcal{T} \mathcal{A}(q)^{\prime}$, where $\mathcal{T}=\operatorname{diag}\left(T_{0}, T_{1}, T_{2}, T_{3}, \ldots\right)$ and $T_{0}=1, T_{n}=T_{n-1} h_{n}(q)$ for $n \geq 1$. Thus this again implies that the Hankel matrix $\mathcal{H}$ is $q-\mathrm{TP}_{r}$ by Lemma 2.4. This proves the claim.

We start to prove that (i) holds. Since

$$
g_{k}(q) g_{k+1}(q) g_{k+2}(q)-h_{k+1}(q) g_{k+2}(q)-g_{k}(q) h_{k+2}(q) \geq_{q} 0
$$

for $k \geq 0$, it follows from Lemma 2.7 (i) and Claim 2.8 that the Hankel matrix $\mathcal{H}$ is $q-\mathrm{TP}_{3}$. So it follows from Proposition 2.5 that $\left\{A_{n, 0}(q)\right\}_{n \geq 0}$ is 2-q-log-convex.

For (ii), it follows from the conditions $g_{k}(q) g_{k+1}(q) \geq_{q} h_{k+1}(q)$ and

$$
\begin{aligned}
& g_{k}(q) g_{k+1}(q) g_{k+2}(q) g_{k+3}(q)-g_{k+2}(q) g_{k+3}(q) h_{k+1}(q)-g_{k}(q)(q) g_{k+3}(q) h_{k+2}(q)- \\
& g_{k}(q) g_{k+1}(q) h_{k+3}(q)+h_{k+1}(q) h_{k+3}(q) \geq_{q} 0
\end{aligned}
$$

for $k \geq 0$ that $\mathcal{J}_{n}(q)$ is $q-\mathrm{TP}_{4}$ by Lemma 2.7 (ii), which again implies that the Hankel matrix $\mathcal{H}$ is $q-\mathrm{TP}_{4}$ by Claim 2.8. Thus, by Proposition 2.5, we have $\left\{A_{n, 0}(q)\right\}_{n \geq 0}$ is 3-q-log-convex.

\section{Applications of Theorem 2.1}

In this section, we will give some applications of Theorem 2.1.

\subsection{Reduced form of Theorem 2.1}

For Theorem 2.1, if let $q$ be a fixed nonnegative real number, then the triangle $\left[A_{n, k}(q)\right]_{n, k \geq 0}$ turns out to be one triangle of nonnegative real numbers. Thus, the next result is a special case of Theorem 2.1. 
Theorem 3.1. Given nonnegative sequences $\left\{g_{n}\right\}_{n \geq 0}$ and $\left\{h_{n}\right\}_{n \geq 0}$, assume that the triangular array $\left[A_{n, k}\right]_{n, k \geq 0}$ satisfies the recurrence

$$
\begin{aligned}
& A_{n, k}=A_{n-1, k-1}+g_{k} A_{n-1, k}+h_{k+1} A_{n-1, k+1}, \\
& A_{n, 0}=g_{0} A_{n-1,0}+h_{1} A_{n-1,1}
\end{aligned}
$$

for $n \geq 1$ and $k \geq 1$, where $A_{0,0}=1, A_{0, k}=0$ for $k>0$.

(i) If $g_{k} g_{k+1} g_{k+2}-h_{k+1} g_{k+2}-g_{k} h_{k+2} \geq 0$ for $k \geq 0$, then the sequence $\left\{A_{n, 0}\right\}_{n \geq 0}$ is 2-log-convex.

(ii) If $g_{k} g_{k+1} \geq h_{k+1}$ and

$$
g_{k} g_{k+1} g_{k+2} g_{k+3}-g_{k+2} g_{k+3} h_{k+1}-g_{k} g_{k+3} h_{k+2}-g_{k} g_{k+1} h_{k+3}+h_{k+1} h_{k+3} \geq 0
$$

for $k \geq 0$, then the sequence $\left\{A_{n, 0}\right\}_{n \geq 0}$ is 3-log-convex.

\subsection{3 -q-log-convexity of generating functions of rows of Riordan arrays}

Riordan arrays are very important in combinatorics. The literature about Riordan arrays is vast and still growing and the applications cover a wide range of subjects, such as enumerative combinatorics, combinatorial sums, recurrence relations and computer science, among other topics [25, 39]. Recall that the Riordan array, denoted by $(g(x), f(x))=$ $\left[R_{n, k}\right]_{n, k \geq 0}$, is an infinite lower triangular matrix whose generating function of the $k$ th column is $x^{k} f^{k}(x) g(x)$ for $k \geq 0$, where $g(0)=1$ and $f(0) \neq 0$. It can also be characterized by two sequences $\left\{a_{n}\right\}_{n \geq 0}$ and $\left\{z_{n}\right\}_{n \geq 0}$ such that

$$
R_{0,0}=1, R_{n+1,0}=\sum_{j \geq 0} z_{j} R_{n, j}, R_{n+1, k+1}=\sum_{j \geq 0} a_{j} R_{n, k+j},
$$

for $n, k \geq 0$.

In fact, there is a close relation between the triangular array $\left[A_{n, k}(q)\right]_{n, k \geq 0}$ in (1.1) and the Riordan arrays. If $g_{k}=g$ and $h_{k}=h$ for $k \geq 1$, then the triangular array $\left[A_{n, k}(q)\right]_{n, k \geq 0}$ turns to be a kind of special interesting Riordan arrays, denoted by $\left[A_{n, k}\right]_{n, k \geq 0}$, which is defined recursively:

$$
\begin{aligned}
& A_{0,0}=1, \quad A_{0, k}=0 \quad(k>0), \\
& A_{n, 0}=e A_{n-1,0}+h A_{n-1,1} \\
& A_{n, k}=A_{n-1, k-1}+g A_{n-1, k}+h A_{n-1, k+1} \quad(n \geq 1) .
\end{aligned}
$$

In fact, the above Riordan arrays $\left[A_{n, k}\right]_{n, k \geq 0}$, or more general triangular array $\left[A_{n, k}(q)\right]_{n, k \geq 0}$ contains many famous combinatorial sequences or classical triangular arrays in combinatorics in a unified approach. The following are several basic examples.

Example 3.1. (1) The Catalan triangle of Aigner [1] is

$$
C=\left[C_{n, k}\right]_{n, k \geq 0}=\left[\begin{array}{cccccc}
1 & & & & & \\
1 & 1 & & & \\
2 & 3 & 1 & & \\
5 & 9 & 5 & 1 & \\
\vdots & & & & \ddots
\end{array}\right]
$$


where $C_{n+1, k}=C_{n, k-1}+2 C_{n, k}+C_{n, k+1}$ and $C_{n+1,0}=C_{n, 0}+C_{n, 1}$. The numbers in the first column are the Catalan numbers $C_{n}$.

(2) The Catalan triangle of Shaprio [40] is

$$
C^{\prime}=\left[C_{n, k}^{\prime}\right]_{n, k \geq 0}=\left[\begin{array}{rrrrrr}
1 & & & & \\
2 & 1 & & & \\
5 & 4 & 1 & & \\
14 & 14 & 6 & 1 & \\
\vdots & & & & \ddots
\end{array}\right]
$$

where $C_{n+1, k}^{\prime}=C_{n, k-1}^{\prime}+2 C_{n, k}^{\prime}+C_{n, k+1}^{\prime}$ for $k \geq 0$. The numbers in the first column are the Catalan numbers $C_{n}$.

(3) The large Schröder triangle [22] is

$$
s=\left[s_{n, k}\right]_{n, k \geq 0}=\left[\begin{array}{rrrrr}
1 & & & & \\
2 & 1 & & & \\
6 & 4 & 1 & & \\
22 & 16 & 6 & 1 & \\
\vdots & & & & \ddots
\end{array}\right],
$$

where $s_{n+1, k}=s_{n, k-1}+2 s_{n, k}+2 s_{n, k+1}$ and $s_{n+1,0}=s_{n, 0}+2 s_{n, 1}$. The numbers in the first column are the large Schröder numbers $S_{n}$.

In fact, we have known that the generating functions of rows in many combinatorial triangles, for example, Narayana triangles of Types $A$ and $B$, the Stirling triangle of the second kind and the Eulerian triangles, have $q$-log-convexity [50] and even stronger 3-qlog-convexity (see Proposition 4.5). Thus, it is natural to consider the similar property of the Riordan arrays. In [52], we stated a criterion for $q$-log-convexity of generating functions of rows of the Riordan array. As an extension, a criterion for 3- $q$-log-convexity can be given as follows.

Theorem 3.2. For $g \geq e \geq 0$ and $h \geq 0$, assume that the Riordan array $\left[A_{n, k}\right]_{n, k \geq 0}$ satisfies the recurrence

$$
\begin{aligned}
& A_{n, k}=A_{n-1, k-1}+g A_{n-1, k}+h A_{n-1, k+1}, \\
& A_{n, 0}=e A_{n-1,0}+h A_{n-1,1},
\end{aligned}
$$

for $n \geq 1$ and $k \geq 1$, where $A_{0,0}=1, A_{0, k}=0$ for $k>0$. Let the generating functions $\mathscr{A}_{n}(q)=\sum_{k \geq 0} A_{n, k} q^{k}$ for $n \geq 0$. If $g e \geq r h$, then $\left\{\mathscr{A}_{n}(q)\right\}_{n \geq 0}$ is $r$-q-log-convex for $r=2,3$ respectively.

Proof. Define a new triangular array $\mathcal{S}=\left[s_{n, k}(q)\right]_{n, k \geq 0}$, where

$$
s_{n, k}(q)=\sum_{i \geq k} A_{n, i} q^{i-k} \text { for all } n \text { and } k .
$$

Thus, by (3.1), we deduce that the triangular array $\mathcal{S}=\left[s_{n, k}(q)\right]_{n, k \geq 0}$ satisfies the recurrence relation

$$
\begin{aligned}
& s_{n, k}(q)=s_{n-1, k-1}(q)+g s_{n-1, k}(q)+h s_{n-1, k+1}(q) \quad(n \geq 1, k \geq 1), \\
& s_{n, 0}(q)=(e+q) s_{n-1,0}(q)+[(g-e) q+h] s_{n-1,1}(q) \quad(n \geq 1),
\end{aligned}
$$


where $s_{0,0}=1, s_{0, k}=0$ for $k>0$. Obviously, $\mathscr{A}_{n}(q)=s_{n, 0}(q)$.

For $r=2$, it follows from $g^{2} \geq g e \geq 2 h$ that

$$
g^{2}(e+q)-[(g-e) q+h] g-(e+q) h=(g e-h) q+e g^{2}-g h-e h \geq_{q} 0
$$

and

$$
g^{3}-h g-g h=g\left(g^{2}-2 h\right) \geq 0 .
$$

Thus we get the 2-q-log-convexity of $\left\{\mathscr{A}_{n}(q)\right\}_{n \geq 0}$ by Theorem 2.1 (i).

For $r=3$, it follows from $g^{2} \geq g e \geq 3 h$ that

$$
\begin{array}{rl} 
& g^{3}(e+q)-[(g-e) q+h] g^{2}-2 g h(e+q)+h[(g-e) q+h] \\
= & {\left[e g^{2}-2 g h+h(g-e)\right] q+g^{3} e-g^{2} h-2 g h e+h^{2}} \\
\geq_{q} & {[g(e g-2 h)+h(g-e)] q+g^{2}(g e-3 h)+h^{2}} \\
\geq_{q} & 0
\end{array}
$$

and

$$
g^{4}-3 h g^{2}+h^{2}=g^{2}\left(g^{2}-3 h\right)+h^{2} \geq 0 .
$$

Hence we obtain the 3-q-log-convexity of $\left\{\mathscr{A}_{n}(q)\right\}_{n \geq 0}$ by Theorem 2.1 (ii). So we complete the proof.

The following result is immediate from Theorem 3.2.

Proposition 3.3. The generating functions of rows in each triangle of Example 3.1 form a 3-q-log-convex sequence.

It is known for the generating function for the sequence $\left\{A_{n, 0}\right\}_{n \geq 0}$ in (3.1) that

$$
\sum_{n \geq 0} A_{n, 0} x^{n}=\frac{1-[2 a-s] x-\sqrt{1-2 s x+\left[s^{2}-4 t\right] x^{2}}}{2[s-a] x+2\left[a^{2}-a s+t\right] x^{2}},
$$

see [3]. Thus, by Theorem 3.2, we have the following criterion for 3-q-log-convexity in terms of the generating functions.

Proposition 3.4. Assume that the generating function

$$
\sum_{n \geq 0} F_{n}(q) x^{n}=\frac{1-[2 a(q)-s(q)] x-\sqrt{1-2 s(q) x+\left[s(q)^{2}-4 t(q)\right] x^{2}}}{2[s(q)-a(q)] x+2\left[a(q)^{2}-a(q) s(q)+t(q)\right] x^{2}},
$$

where polynomials $s(q) \geq_{q} a(q) \geq_{q} 0$ and $t(q) \geq_{q} 0$. Then we have the following results:

(i) If a $(q) s(q) \geq_{q} 2 t(q)$, then the sequence $\left\{F_{n}(q)\right\}_{n \geq 0}$ is 2-q-log-convex.

(ii) If $a(q) s(q) \geq_{q} t(q)$ and $a(q) s^{3}(q)-2 a(q) s(q) t(q)-s^{2}(q) t(q)+t^{2}(q) \geq_{q} 0$, then the sequence $\left\{F_{n}(q)\right\}_{n \geq 0}$ is 3-q-log-convex.

\section{Applications of Theorem 2.2}

In this section, we will apply Theorem 2.2 to some classical examples in combinatorics. 


\section{$4.1 \quad 2-q$-log-convexity of alternating Eulerian polynomials}

Let $\mathcal{S}_{n}$ denote the symmetric group of all permutations of $[n]$. Similar to the descent statistic of $\mathcal{S}_{n}$, the number of alternating descents of a permutation $\pi \in \mathcal{S}_{n}$ is defined by

$$
\operatorname{altdes}(\pi)=|\{2 i: \pi(2 i)<\pi(2 i+1)\} \cup\{2 i+1: \pi(2 i+1)>\pi(2 i+2)\}| .
$$

We say that $\pi$ has a 3 -descent at index $i$ if $\pi(i) \pi(i+1) \pi(i+2)$ has one of the patterns: 132 , 213 , or 321. Chebikin [14] proved that the alternating descent statistic of permutations in $\mathcal{S}_{n}$ is equidistributed with the 3 -descent statistic of permutations in $\left\{\pi \in \mathcal{S}_{n+1}: \pi_{1}=1\right\}$. Then alternating Eulerian polynomials $A_{n}^{*}(x)$ and the alternating Eulerian numbers $A_{n, k}^{*}$ are defined as follows:

$$
A_{n}^{*}(x)=\sum_{\pi \in \mathcal{S}_{n}} x^{\operatorname{altdes}(\pi)}=\sum_{k=0}^{n-1} A_{n, k}^{*} x^{k}
$$

where the first few items $A_{1}^{*}(x)=1, A_{2}^{*}(x)=1+x$ and $A_{3}^{*}(x)=2+2 x+2 x^{2}$. It was proved that

$$
\sum_{n \geq 1} A_{n}^{*}(x) \frac{z^{n}}{n !}=\frac{\sec (1-x) z+\tan (1-x) z-1}{1-x[\sec (1-x) z+\tan (1-x) z]}
$$

Proposition 4.1. Let $A_{n}^{*}(q)$ be the alternating Eulerian polynomials for $n \geq 0$. Then

(i) we have

$$
\sum_{n=0}^{\infty} A_{n+1}^{*}(q) x^{n}=\frac{1}{1-g_{0} x-\frac{h_{1} x^{2}}{1-g_{1} x-\frac{h_{2} x^{2}}{1-g_{2} x-\ldots}}},
$$

where $g_{i}=(i+1)(q+1)$ and $h_{i}=\left(q^{2}+1\right) i(i+1) / 2$ for $i \geq 0$.

(ii) The sequence $\left\{A_{n+1}^{*}(q)\right\}_{n \geq 0}$ is 2-q-log-convex.

Proof. Let $D_{x}$ denote the differential operator $\frac{d}{d x}$. In 1995, Hoffman considered derivative polynomials defined respectively by $D_{x}^{n}(\tan (x))=P_{n}(\tan (x))$ for $n \geq 0$, where $P_{0}(q)=q$ and $P_{n+1}(q)=\left(1+q^{2}\right) P_{n}^{\prime}(q)$. In [44], it was proved that the generating function of $P_{n}(q)$ has the following continued fraction expansion:

$$
\sum_{n=0}^{\infty} \frac{P_{n+1}(q)}{1+q^{2}} x^{n}=\frac{1}{1-g_{0} x-\frac{h_{1} x^{2}}{1-g_{1} x-\frac{h_{2} x^{2}}{1-g_{2} x-\ldots}}}
$$

where $g_{i}=2(i+1) q$ and $h_{i}=\left(1+q^{2}\right) i(i+1)$ for $i \geq 0$.

On the other hand, Ma and Yeh [31] recently proved

$$
2^{n}\left(1+x^{2}\right) A_{n}^{*}(x)=(1-x)^{n+1} P_{n}\left(\frac{1+x}{1-x}\right)
$$


for $n \geq 1$. Thus by (4.1), we have

$$
\begin{aligned}
& \sum_{n=0}^{\infty} 2^{n} A_{n+1}^{*}(q)(x / 2)^{n} \\
= & \sum_{n=0}^{\infty} \frac{(1-q)^{n+2}}{2\left(1+q^{2}\right)} P_{n+1}\left(\frac{1+q}{1-q}\right)(x / 2)^{n} \\
= & \sum_{n=0}^{\infty} \frac{P_{n+1}\left(\frac{1+q}{1-q}\right)}{1+\left(\frac{1+q}{1-q}\right)^{2}}\left[\frac{(1-q) x}{2}\right]^{n} \\
= & \frac{1-g_{0} x-\frac{h_{1} x^{2}}{1-g_{1} x-\frac{h_{2} x^{2}}{1-g_{2} x-\ldots}}}{1-\ldots}
\end{aligned}
$$

where $g_{i}=(i+1)(q+1)$ and $h_{i}=\left(q^{2}+1\right) i(i+1) / 2$ for $i \geq 0$. This proves that (i) holds.

(ii) Because $g_{i}=(i+1)(q+1)$ and $h_{i}=\left(q^{2}+1\right) i(i+1) / 2$ for $i \geq 0$, we have

$$
\begin{array}{rl} 
& g_{i}(q) g_{i+1}(q) g_{i+2}(q)-h_{i+1}(q) g_{i+2}(q)-g_{i}(q) h_{i+2}(q) \\
= & (i+3)(i+2)(i+1)(1+q)^{3}-(1+q)\left(q^{2}+1\right)(i+3)(i+2)(i+1) / 2 \\
& -(q+1)\left(q^{2}+1\right)(i+3)(i+2)(i+1) / 2 \\
= & 2 q(q+1)(i+3)(i+2)(i+1) \\
\geq_{q} & 0
\end{array}
$$

Thus, by Theorem 2.2 (i), we obtain that $\left\{A_{n}^{*}(q)\right\}_{n \geq 1}$ is 2- $q$-log-convex.

Remark 4.2. For $\left\{A_{n}^{*}(q)\right\}_{n \geq 1}$, it is easy to get that the condition (ii) in Theorem 2.2 does not follow. In fact, $\left[A_{i+j}^{*}(q)\right]_{i, j \geq 1}$ is not $q-T P_{4}$ because

$$
\left|\begin{array}{llll}
A_{2}^{*}(q) & A_{3}^{*}(q) & A_{4}^{*}(q) & A_{5}^{*}(q) \\
A_{3}^{*}(q) & A_{4}^{*}(q) & A_{5}^{*}(q) & A_{6}^{*}(q) \\
A_{4}^{*}(q) & A_{5}^{*}(q) & A_{6}^{*}(q) & A_{7}^{*}(q) \\
A_{5}^{*}(q) & A_{6}^{*}(q) & A_{7}^{*}(q) & A_{8}^{*}(q)
\end{array}\right|=-324+1296 q+2592 q^{2}+\cdots+1296 q^{15}-324 q^{16} .
$$

But it seems that $\left\{A_{n}^{*}(q)\right\}_{n \geq 1}$ is still 3-q-log-convex by the computation from Mathematica.

\subsection{2-log-convexity of Euler numbers}

Recall the definition of Euler numbers as follows. A permutation $\pi$ of the $n$-element set $[n]=\{1,2, \ldots, n\}$ is alternating if $\pi(1)>\pi(2)<\pi(3)>\pi(4)<\cdots \pi(n)$. The number $E_{n}$ of alternating permutations of $[n]$ is known as an Euler number. The sequence has the exponential generating function

$$
\sum_{k=0}^{n} E_{n} \frac{x^{n}}{n !}=\tan x+\sec x .
$$

Liu and Wang [30] proved that $\left\{E_{n}\right\}_{n \geq 0}$ is log-convex by Davenport-Pólya Theorem. The next result strengthens the log-convexity of Euler numbers. 
Proposition 4.3. The Euler numbers form a 2-log-convex sequence.

Proof. In [44], it was proved that

$$
\sum_{n=0}^{\infty} E_{n+1} x^{n}=\frac{1}{1-g_{0} x-\frac{h_{1} x^{2}}{1-g_{1} x-\frac{h_{2} x^{2}}{1-g_{2} x-\ldots}}},
$$

where $g_{i}=i+1$ and $h_{i}=i(i+1) / 2$ for $i \geq 0$. Note that

$$
g_{i} g_{i+1} g_{i+2}-h_{i+1} g_{i+2}-g_{i} h_{i+2}=0
$$

for $i \geq 0$. Hence the 2-log-convexity of $\left\{E_{n+1}\right\}_{n \geq 0}$ follows from Theorem 2.2 (i).

Remark 4.4. For $\left\{E_{n+1}\right\}_{n \geq 0}=\{1,2,5,16,61,272,1385,7936,50521,353792, \cdots\}$, then

$$
g_{k} g_{k+1} g_{k+2} g_{k+3}-g_{k+2} g_{k+3} h_{k+1}-g_{k} g_{k+3} h_{k+2}-g_{k} g_{k+1} h_{k+3}+h_{k+1} h_{k+3}=-6
$$

for $k=0$ and

$$
\left|\begin{array}{cccc}
E_{n} & E_{n+1} & E_{n+2} & E_{n+3} \\
E_{n+1} & E_{n+2} & E_{n+3} & E_{n+4} \\
E_{n+2} & E_{n+3} & E_{n+4} & E_{n+5} \\
E_{n+3} & E_{n+4} & E_{n+5} & E_{n+6}
\end{array}\right|=-324,-154224
$$

for $n=2,4$, respectively. Thus the condition (ii) in Theorem 2.2 does not follow and we can't get 3-log-convexity of $\left\{E_{n+1}\right\}_{n \geq 0}$. But it seems that $\left\{E_{n+1}\right\}_{n \geq 0}$ is still 3-log-convex by the computation from Mathematica.

\section{$4.3 \quad 3-q$-log-convexity of classical combinatorial polynomials}

It is known that many important combinatorial polynomials, such as the Bell polynomials, Eulerian polynomials, and Narayana polynomials of types A and B, and so on, are $q$-logconvex. By Theorem 2.1, we have the following stronger result in a unified approach.

Proposition 4.5. The six sequences of polynomials in Example 1.1 are all 3-q-log-convex.

Proof. Because the proofs are similar, for brevity, we only present the proof of the 3-qlog-convexity of the Bell polynomials and omit the proofs of others.

Notice for the case of the Bell polynomials that $g_{k}(q)=q+k$ and $h_{k}(q)=k q$ for $k \geq 0$. It is obvious that

$$
g_{k}(q) g_{k+1}(q)-h_{k+1}(q)=q^{2}+k q+k^{2}+k \geq_{q} 0 .
$$

Thus, in order to prove the 3-q-log-convexity of the Bell polynomials, by Theorem 2.1 (ii), it suffices to check

$$
\begin{array}{rl} 
& g_{k}(q) g_{k+1}(q) g_{k+2}(q) g_{k+3}(q)-g_{k+2}(q) g_{k+3}(q) h_{k+1}(q)-g_{k}(q) g_{k+3}(q) h_{k+2}(q) \\
& -g_{k}(q) g_{k+1}(q) h_{k+3}(q)+h_{k+1}(q) h_{k+3}(q) \\
= & k^{4}+(6+q) k^{3}+\left(11+3 q+q^{2}\right) k^{2}+\left(q^{3}+q^{2}+2 q+6\right) k+q^{4} \\
\geq_{q} & 0
\end{array}
$$

as desired. This proof is complete. 
Remark 4.6. In fact, the Hankel matrix $\left[A_{i+j, 0}(q)\right]_{i, j \geq 0}$ for each sequence of polynomials in Example 1.1 are q-TP [48]. So we can also obtain the 3-q-log-convexity by Proposition 2.5.

\subsection{3- $q$-log-convexity from the Stieltjes continued fractions}

Let $\left\{S_{n}(q)\right\}_{n \geq 0}$ be a sequence of polynomials with $S_{0}(q)=1$. The expansion

$$
\sum_{i=0}^{\infty} S_{n}(q) x^{i}=\frac{1}{1-\frac{t_{1}(q) x}{1-\frac{t_{2}(q) x}{1-\ldots}}}
$$

is called the Stieltjes continued fraction. In view of the following famous contraction formulae

$$
\frac{1}{1-\frac{c_{1} x}{1-\frac{c_{2} x}{1-\ldots}}}=\frac{1}{1-c_{1} x-\frac{c_{1} c_{2} x^{2}}{1-\left(c_{2}+c_{3}\right) x-\frac{c_{3} c_{4} x^{2}}{1-\ldots}}},
$$

we deduce the Jacobi continued fraction expansion

$$
\sum_{i=0}^{\infty} S_{n}(q) x^{i}=\frac{1}{1-t_{1}(q) x-\frac{t_{1}(q) t_{2}(q) x^{2}}{1-\left[t_{2}(q)+t_{3}(q)\right] x-\frac{t_{3}(q) t_{4}(q) x^{2}}{1-\ldots}}} .
$$

Theorem 4.7. If $t_{n}(q)$ are polynomials in $q$ for $n \geq 1$ and $t_{n}(q) \geq_{q} 0$, then the sequence $\left\{S_{n}(q)\right\}_{n \geq 0}$ defined in (4.2) is 3-q-log-convex.

Proof. Noting that $g_{i}=t_{2 i+1}(q)+t_{2 i}(q)$ and $h_{i}=t_{2 i-1}(q) t_{2 i}(q)$, we obtain

$$
g_{i} g_{i+1}-h_{i+1}=t_{1+2 i}(q) t_{3+2 i}(q)+t_{2 i}(q) t_{2+2 i}(q)+t_{2 i}(q) t_{3+2 i}(q) \geq_{q} 0
$$

and

$$
\begin{array}{rl} 
& g_{i} g_{i+1} g_{i+2} g_{i+3}-g_{i+2} g_{i+3} h_{i+1}-g_{i} g_{i+3} h_{i+2}-g_{i} g_{i+1} h_{i+3}+h_{i+1} h_{i+3} \\
= & t_{2 i}(q)\left[t_{3+2 i}(q) t_{5+2 i}(q) t_{7+2 i}(q)+t_{2+2 i}(q)\left[t_{5+2 i}(q) t_{7+2 i}(q)+t_{4+2 i}(q)\left(t_{6+2 i}(q)+t_{7+2 i}(q)\right)\right]\right] \\
& +t_{1+2 i}(q) t_{3+2 i}(q) t_{5+2 i}(q) t_{7+2 i}(q) \\
\geq_{q} & 0
\end{array}
$$

Thus, by Theorem 2.2, the sequence $\left\{S_{n}(q)\right\}_{n \geq 0}$ defined in (4.2) is 3- $q$-log-convex.

Remark 4.8. In fact, by [48, Lemma 3.3], the Hankel matrix $\left[S_{i+j, 0}(q)\right]_{i, j \geq 0}$ for the sequence $\left\{S_{n}(q)\right\}_{n \geq 0}$ defined in (4.2) is q-TP. That is to say that $\left\{S_{n}(q)\right\}_{n \geq 0}$ defined in (4.2) is a q-Stieltjes sequences. For $q$ being nonnegative real numbers, this particularly gives a new proof of Stieltjes sequences. 
The elliptic functions $c n$ and $d n$ are defined by

$$
\operatorname{cn}(u, \alpha)=\operatorname{cosam}(u, \alpha) ; \quad d n(u, \alpha)=\sqrt{1-\alpha^{2} \sin ^{2} a m(u, \alpha)},
$$

where $\operatorname{am}(u, \alpha)$ is the inverse of an elliptic integral: by definition

$$
a m(u, \alpha)=\phi \quad \text { iff } \quad u=\int_{0}^{\phi} \frac{d t}{\sqrt{1-\alpha^{2} \sin ^{2} t}} .
$$

The function $c n(u, \alpha)$ and $d n(u, \alpha)$ have power series expansions:

$$
\begin{aligned}
& c n(u, \alpha)=\sum_{n \geq 0}(-1)^{n-1} c_{n}\left(\alpha^{2}\right) \frac{u^{2 u}}{2 n !} \\
& d n(u, \alpha)=\sum_{n \geq 0}(-1)^{n-1} d_{n}\left(\alpha^{2}\right) \frac{u^{2 u}}{2 n !}
\end{aligned}
$$

where $c_{n}(q)$ and $d_{n}(q)$ are polynomials of degree $n-1$ with $d_{n}(q)$ the reciprocal polynomial of $c_{n}(q)$. In addition, Flajolet [23, Theorem 4] proved that the coefficient $c_{n, k}$ of the polynomial $c_{n}(q)$ counts the alternating permutations over $[2 n]$ having $k$ minima of even value and

$$
\sum_{i=0}^{\infty} c_{n}(q) x^{i}=\frac{1}{1-\frac{1^{2} x}{1-\frac{2^{2} q x}{1-\frac{3^{2} x}{1-\frac{4^{2} q x}{\cdots}}}}} .
$$

Thus the following proposition is an immediate consequence of Theorem 4.7.

Proposition 4.9. The polynomials $c_{n}(q)$ form a 3-q-log-convex sequence.

\section{Concluding remarks and open problems}

In this paper we have given some sufficient conditions for the 2-q-log-convexity and $3-q-\log$-convexity. Finally, using our results we show the 2-q-log-convexity or 3-q-logconvexity of more classical combinatorial sequences of polynomials and numbers.

Based on the result in Theorem 2.2, it is natural to consider the following problem for the infinite $q$-log-convexity of Boros-Moll polynomial [15].

Problem 5.1. Can we obtain a continued fraction expansion for the generating function of the sequence of Boros-Moll polynomials?

By Proposition 4.5, we have known that each sequence of polynomials in Example 1.1 is 3-q-log-convex. In addition, for any fixed nonnegative real number $q$, each sequence of polynomials in Example 1.1 is infinitely log-convex [48]. Thus, we conclude this paper with the following conjecture concerning the infinite $q$-log-convexity for further research. 
Conjecture 5.2. Every sequence of polynomials in Example 1.1 is infinitely q-log-convex.

Note a criterion for infinite log-convexity that if the infinite Hankel matrix $\left[x_{i+j}\right]_{i, j \geq 0}$ is TP, then so is $\left[x_{i+j+2} x_{i+j}-x_{i+j+1}^{2}\right]_{i, j \geq 0}$, see [48]. Thus, Proposition 2.5 appears to indicate the following conjecture, which is a possible approach to the higher order $q$-log-convexity.

Conjecture 5.3. Let $r$ be any positive integer and $\left\{x_{n}(q)\right\}_{n \geq 0}$ be a sequence of polynomials. If the infinite Hankel matrix $\left[x_{i+j}(q)\right]_{i, j \geq 0}$ is $q-T P_{r+1}$, then

$$
\left[x_{i+j+2}(q) x_{i+j}(q)-x_{i+j+1}(q)^{2}\right]_{i, j \geq 0}
$$

is $q-T P_{r}$.

This Conjecture 5.3 may be proved by the combinatorial approach from the planar network, see $[11,24,37,42]$ for details.

\section{Acknowledgements}

The author would like to thank the anonymous reviewer for many valuable remarks and suggestions to improve the original manuscript. In addition, results of this paper were presented in the Institute of Mathematics, Academia Sinica, Taipei in Jan. 2016, the seventh National Conference on Combinatorics and Graph Theory at Hebei Normal University in Aug. 2016 and at Nakai University in Oct. 2016, respectively.

\section{References}

[1] M. Aigner, Catalan-like numbers and determinants, J. Combin. Theory, Ser. A 87 (1999) 33-51.

[2] M. Aigner, Catalan and other numbers-a recurrent theme, in: H. Crapo, D. Senato (Eds.), Algebraic Combinatorics and Computer Science, Springer, Berlin, 2001, pp. $347-390$.

[3] M. Aigner, Enumeration via ballot numbers, Discrete Math. 308 (2008) 2544-2563.

[4] F. Ardila, M. Beck, S. Hosten, J. Pfeifle, K. Seashore, Root polytopes and growth series of root lattices, SIAM J. Discrete Math. 25(1) (2011) 360-378.

[5] M. Benson, Growth series of finite extensions of $Z_{n}$ are rational, Invent. Math. 73 (1983) 251-269.

[6] J. Bonin, L. Shapiro and R. Simion, Some q-analogues of the Schröder numbers arising from combinatorial statistics on lattice paths, J. Statist. Plann. Inference 34 (1993) 35-55.

[7] G. Boros and V. Moll, Irresistible integrals, Cambridge University Press, Cambridge, 2004. Symbolics, analysis and experiments in the evaluation of integrals. 
[8] P. Brändrén, Iterated sequeces and the geometry of zeros, J. Reine Angew. Math 658 (2011) 115-131.

[9] F. Brenti, Unimodal, log-concave, and Pólya frequency sequences in combinatorics, Mem. Amer. Math. Soc. 413 (1989).

[10] F. Brenti, Log-concave and unimodal sequences in algebra, combinatorics, and geometry: an update, Contemp. Math. 178 (1994) 71-89.

[11] F. Brenti, Combinatorics and total positivity, J. Combin. Theory Ser. A 71 (1995) $175-218$.

[12] F. Brenti, The applications of total positivity to combinatorics, and conversely, Total positivity and its applications (Jaca, 1994), 451-473, Math. Appl., 359, Kluwer Acad. Publ., Dordrecht, 1996.

[13] L.M. Butler, The $q$-log concavity of $q$-binomial coeffcients, J. Combin. Theory Ser. A 54 (1990) 54-63.

[14] D. Chebikin, Variations on descents and inversions in permutations, Electron. J. Combin. 15 (2008), R132.

[15] W.Y.C. Chen, Log-concavity and q-Log-convexity Conjectures on the Longest Increasing Subsequences of Permutations, arXiv: 0806.3392v2.

[16] W.Y.C. Chen, D.Q.J. Dou, A.L.B. Yang, Brändén's Conjectures on the Boros-Moll Polynomials, International Mathematics Research Notices, 20 (2013) 4819-4828.

[17] W.Y.C. Chen, R.L. Tang, L.X.W. Wang, A.L.B. Yang, The $q$-log-convexity of the Narayana polynomials of type B, Adv. in Appl. Math. 44(2) (2010) 85-110.

[18] W.Y.C. Chen, L.X.W. Wang, A.L.B. Yang, Schur positivity and the $q$-log-convexity of the Narayana polynomials, J. Algebraic Combin. 32(3) (2010) 303-338.

[19] W.Y.C. Chen, L.X.W. Wang and A.L.B. Yang, Recurrence relations for strongly $q$-log-convex polynomials, Canad. Math. Bull. 54 (2011) 217-229.

[20] W.Y.C. Chen, E.X.W. Xia, 2-log-concavity of the Boros-Moll polynomials, Proc. Edinburgh Math. Soc. (2) 56(3) (2013) 701-722.

[21] W.Y.C. Chen, E.X.W. Xia, The 2-log-convexity of the Apéry numbers. Proc. Amer. Math. Soc. 139 (2) (2011) 391-400.

[22] G.-S. Cheon, H. Kim and L.W. Shapiro, Combinatorics of Riordan arrays with identical A and Z sequences, Discrete Math. 312 (2012) 2040-2049.

[23] P. Flajolet, Combinatorial aspects of continued fractions, Discrete Math. 32 (1980) $125-161$.

[24] I. Gessel and G. Viennot, Binomial determinants, path, and hook length formulae, Adv. Math. 58 (1985) 300-321. 
[25] T.-X. He, Parametric Catalan numbers and Catalan triangles, Linear Algebra Appl. 438 (2013) 1467-1484.

[26] S. Karlin, Total Positivity, Vol. I, Stanford University Press, Stanford, 1968.

[27] M. Kauers and P. Paule, A computer proof of Moll's log-concavity conjecture, Proc. Amer. Math. Soc. 135 (2007) 3847-3856.

[28] P. Leroux, Reduced matrices and $q$-log concavity properties of $q$-Stirling numbers, J. Combin. Theory Ser. A 54 (1990) 64-84.

[29] Z. Lin, J. Zeng, Positivity properties of Jacobi-Stirling numbers and generalized Ramanujan polynomials, Adv. in Appl. Math. 53 (2014) 12-27.

[30] L.L. Liu, Y. Wang, On the log-convexity of combinatorial sequences, Adv. in. Appl. Math. 39 (2007) 453-476.

[31] S.-M Ma, Y.-N. Yeh, Enumeration of permutations by number of alternating descents, Discrete Math 339 (2016) 1362-1367.

[32] V.H. Moll, The evaluation of integrals: A personal story, Notices Amer. Math. Soc. 49 (2002) 311-317.

[33] P.R.W. McNamara, B.E. Sagan, Infinite log-concavity: developments and conjectures, Adv. in. Appl. Math. 44 (2010) 1-15.

[34] A. Pinkus, Totally Positive Matrices, Cambride University Press Cambridge, 2010.

[35] V. Reiner, Noncrossing partitions for classical reflection groups, Discrete Math. 177 (1992) 195-222.

[36] S. Roman, The Umbral Calculus, Academic Press, New York, 1984.

[37] B.E. Sagan, Log concave sequences of symmetric functions and analogs of the JacobiTrudi determinants, Trans. Amer. Math. Soc. 329 (1992) 795-811.

[38] B.E. Sagan, Unimodality and the reflection principle, Ars Combin. 48 (1998) 65-72.

[39] L.W. Shapiro, S. Getu, W.-J. Woan, L.C. Woodson, The Riordan group, Discrete Appl. Math. 34(1-3) (1991) 229-239.

[40] L.W. Shapiro, A Catalan triangle, Discrete Math. 14 (1976) 83-90.

[41] R.P. Stanley, Log-concave and unimodal sequences in algebra, combinatorics, and geometry, Ann. New York Acad. Sci. 576 (1989) 500-534.

[42] J. R. Stembridge, Nonintersecting paths, pfaffians, and plane partitions, Adv. Math. 83 (1990) 96-131.

[43] R. Sulanke, The Narayana distribution, J. Statist. Plann. Inference 101 (2002) 311326. 
[44] M. Josuat-Vergès, Enumeration of snakes and cycle-alternating permutations, Austraian J. Combin. 60(3) (2014) 279-305.

[45] H.S. Wall, Analytic Theory of Continued Fractions, Van Nostrand, Princeton, NJ, 1948.

[46] Y. Wang and Y.-N. Yeh, Polynomials with real zeros and Pólya frequency sequences, J. Combin. Theory Ser. A 109 (2005) 63-74.

[47] Y. Wang and Y.-N. Yeh, Log-concavity and LC-positivity, J. Combin. Theory Ser. A 114 (2007) 195-210.

[48] Y. Wang, B.-X. Zhu, Log-convex and Stieltjes moment sequences, Adv. in Appl. Math. 81 (2016) 115-127.

[49] B.-X. Zhu, H. Sun, Linear transformations preserving the strong $q$-log-convexity of polynomials, Electron. J. Combin. 22(3) (2015) $\sharp \mathrm{P} 3.27$.

[50] B.-X. Zhu, Log-convexity and strong $q$-log-convexity for some triangular arrays, Adv. in Appl. Math. 50 (2013) 595-606.

[51] B.-X. Zhu, Some positivities in certain triangular arrays, Proc. Amer. Math. Soc. 142 (2014) 2943-2952.

[52] B.-X. Zhu, Log-concavity and strong q-log-convexity for Riordan arrays and recursive matrices, Proc. Roy. Soc. Edinburgh Sect. A 147 (2017) 1297-1310. 